\title{
Des politiques publiques aux programmes : l'évaluation sauvée par la LOLF ? Les enseignements de la politique de la ville
}

\author{
Renaud Epstein*
}

Publié dans la Revue Française des Affaires Sociales, juin 2010, n¹-2, p.227-250.

Résumé:

En faisant basculer les politiques de l'Etat dans une logique de programme et de gestion par la performance, la loi organique relative aux lois de finances du 1er août 2001 créerait les conditions d'un renouveau évaluatif, mettant fin aux errements de l'évaluation des politiques publiques des années 1990. L'entrée en application du texte organique est trop récente pour évaluer précisément ses effets en la matière. L'examen de la politique de la ville et de son évaluation conduit néanmoins à s'interroger sur la validité des discours néo-managériaux en vogue actuellement, qui présentent le passage à une logique de programme comme une avancée décisive pour l'évaluation et, au-delà, pour la performance publique. Non seulement parce qu'il ne suffit de créer les conditions de l'évaluabilité d'un programme pour que celui-ci soit évalué, mais aussi parce que les transformations institutionnelles conduites à cette fin s'avèrent fort peu favorables à l'émergence d'un débat pluraliste.

L'évaluation des politiques publiques a connu un indéniable essor au cours des années 1990 sous l'effet combiné de la politique de modernisation de l'Etat initiée par Michel Rocard, de la multiplication des contractualisations territoriales et du poids croissant des programmes européens dans les politiques publiques françaises. La plupart des ministères, des grandes collectivités territoriales et des services publics se sont dotés de services d'évaluation. Les démarches évaluatives se sont systématisées ${ }^{1}$, ses acteurs se sont professionnalisés, et un marché de l'évaluation et de la formation à cette pratique s'est structuré.

Ce mouvement de développement et d'institutionnalisation de l'évaluation dans l'action publique française est d'autant plus remarquable que cette pratique s'est heurtée à de très nombreux freins qui en ont fortement réduit la portée ${ }^{2}$. Un quart de siècle après son apparition ${ }^{3}$, l'évaluation des politiques publiques présente un bilan mitigé. Le dispositif d'évaluation interministériel mis en place par le décret du 22 janvier 1990, organisé autour d'un Comité interministériel de l'évaluation, d'un Conseil scientifique de l'évaluation et d'un Fonds national de développement de l'évaluation a été mis en sommeil douze ans plus tard. Les évaluations conduites dans ce cadre n'ont eu qu'un écho limité dans le débat public et rares sont celles qui ont eu des effets sensibles sur les décisions politiques (Perret, 2001). Le bilan n'est guère plus probant à

\footnotetext{
* Maître de conférences en sciences politiques - Université de Nantes

Chercheur associé à l'Institut des Sciences sociales du Politique (ENS Cachan - CNRS)

${ }^{1}$ A l'occasion du congrès 2008 de la Société française de l'évaluation, le secrétaire d'Etat chargé de la prospective et de l'évaluation des politiques publiques a déclaré que les administrations d'Etat réalisaient près de 700 évaluations chaque année (cité par Perret, 2008).

${ }^{2}$ Pour une analyse de ces freins, le lecteur se reportera au dossier « L'évaluation de l'action publique : pour quand ? » publié par la revue Politiques et management public en 2002 (vol.20, $n^{\circ} 4$ ), notamment l'article introductif de Patrice Duran ainsi que ceux de Véronique Chanut et Jean-Claude Thoenig.

${ }^{3}$ On peut en effet considérer que le colloque international sur l'évaluation des politiques publiques organisé en décembre 1983 par la direction de la Prévision du ministère de l'Economie, des Finances et du Budget constitue la scène originelle de l'évaluation française. Les actes du colloque ont été publiés l'année suivante (Nioche, Poinsard, 1984)
} 
l'échelon infranational. Certes, les démarches évaluatives qui se sont multipliées dans les régions, les départements et les villes n'ont pas été inutiles. Elles ont contribué au pilotage des politiques locales, en particulier de celles conduites dans un cadre partenarial, en mettant en place des instruments de suivi des politiques menées, d'observation de leurs objets et d'écoute de leurs bénéficiaires, ou en instaurant de scènes de débat technique qui ont contribué à l'apprentissage collectif et à la résolution des conflits entre leurs acteurs (Spenlehauer, Warin, 2000). Mais il s'agit là d'apports sinon mineurs, tout du moins limités au regard des ambitions initiales, formulées en termes de démocratisation et d'amélioration de l'efficacité de la gestion publique.

En recourant au jargon évaluatif, on pourrait donc dire que la politique française d'évaluation peut se prévaloir d'une certaine effectivité, mais n'apparaît guère probante sur les registres de l'efficacité, de l'efficience et de l'impact. A écouter les discours des acteurs actuellement en pointe dans la promotion de l'évaluation -à savoir les hauts fonctionnaires du ministère du Budget, désormais en position dominante pour la conduite de la réforme de l'Etat (Bezes, 2009) - cet échec relatif de l'évaluation tiendrait en grande partie à l'option initialement retenue en France, consistant à prendre pour objet des politiques publiques, à la différence de nombreux pays qui ont privilégié l'évaluation de programmes.

Dans le Petit guide de l'évaluation des politiques publiques publié en 1996, le Conseil Scientifique de l'Evaluation distinguait nettement les notions de politique et de programme: "Le terme programme désigne, en principe, une séquence d'actions, limitée dans le temps et surtout définie précisément dans ses moyens et ses objectifs opératoires [...] La notion de politique, quant à elle, renvoie plutôt à un ensemble complexe de programmes, procédures et régulations concourant à un même objectif général »" Le choix de l'évaluation des politiques publiques était assurément cohérent avec le mode de structuration de l'action publique française, mais il ajoutait tout aussi assurément un degré de complexité à l'exercice évaluatif, obligeant ses acteurs à démêler l'écheveau complexe des programmes, des procédures, des lois et règlements, des actions et des financements contribuant à une même politique publique.

La loi organique relative aux lois de finances (LOLF) du 1'er août 2001 a changé la donne évaluative. Le budget de l'État, qui était jusqu'alors découpé par ministère et par nature, est désormais structuré en missions et programmes. Cette nouvelle présentation budgétaire, qui permet d'identifier précisément les moyens affectés à un programme donné, s'accompagne d'une clarification des objectifs poursuivis: chaque programme s'accompagne d'un projet annuel de performance, organisé autour d'un nombre restreint d'objectifs (trois à six) assortis d'indicateurs de performance. En organisant le basculement des politiques de l'Etat dans une logique de programme et de gestion par la performance, le réforme budgétaire semble ainsi créer les conditions d'un renouveau évaluatif (Siné, Veillet, 2007), mettant fin aux errements antérieurs de l'évaluation des politiques publiques : celle-ci se concentrait sur les processus de l'action publique, en laissant le plus souvent de côté ses résultats, et ne suscitait de ce fait qu'un intérêt limité chez les responsables politiques. L'évaluation peut désormais porter sur des programmes, et les responsables administratifs qui en ont la charge doivent rendre compte chaque année de leurs résultats devant le Parlement.

La LOLF n'est pleinement entrée en application qu'en 2006. II est donc trop tôt pour juger globalement des transformations induites par cette réforme majeure, qui produit des effets en chaîne sur l'action publique. L'exercice consistant à tirer des enseignements généraux d'un cas singulier est toujours hasardeux. On peut néanmoins, à partir de l'examen de la politique de la ville et de son évaluation, ébaucher des pistes de réflexion plus générale sur l'évaluation des politiques publiques. Car la politique de la ville a joué un rôle particulier dans l'histoire de l'évaluation française, ce qui lui confère une fonction heuristique en la matière. Aujourd'hui encore, elle fournit un éclairage utile sur les évolutions de l'évaluation, la réforme opérée par la loi d'orientation et de programmation pour la politique de la ville et la rénovation urbaine du $1^{\text {er }}$ août 2003 (dite loi Borloo) ayant organisé sa conformation anticipée à « l'esprit de la LOLF ». On s'interrogera donc, après avoir retracé l'histoire de l'évaluation de la politique de la ville, sur la validité des discours néo-

\footnotetext{
${ }^{4}$ S'il existe une différence théorique entre les notions de politique et de programme, il est cependant souvent difficile de distinguer dans la pratique les deux concepts (Kessler et al., 1998).
} 
managériaux qui présentent le passage à une logique de programme comme une avancée décisive pour l'évaluation.

\section{Retour sur 25 ans d'évaluation de la politique de la ville}

La politique de la ville a servi de terrain privilégié pour l'expérimentation des premières démarches évaluatives dans les années 1980 (Ion, Peroni, Roux, 1996), puis de cheval de Troie pour la diffusion de cette pratique dans les appareils administratifs au cours de la décennie suivante. De toutes les politiques publiques françaises, il s'agit sans doute possible de celle qui a fait l'objet du plus grand nombre d'évaluations tant à l'échelle nationale que régionale et locale. Dans certains sites phares de la politique de la ville, il n'est pas rare de retrouver une demi-douzaine de rapports évaluant sa mise en œuvre locale, couvrant l'ensemble de son histoire. Si l'on additionne l'ensemble des évaluations conduites aux différents niveaux au cours des vingt cinq dernières années, le bilan est impressionnant, avec plus de 300 rapports d'évaluation qui sommeillent dans les placards administratifs ${ }^{5}$.

Le lien intime unissant la politique de la ville et l'évaluation des politiques publiques s'est affirmé dès le stade de leur gestation. Les conventions Habitat et vie sociale signées à partir de 1977 n'ont pas seulement servi de creuset pour l'ébauche des principes structurants de la politique de la ville (transversalité, partenariat, participation des habitants) et de ses programmes d'actions, articulant réhabilitation des immeubles dégradés, développement de l'offre de services collectifs et soutien aux initiatives des forces vives des quartiers populaires réunies dans les associations. Elles ont aussi servi de cadre pour l'expérimentation des premières démarches d'évaluation locale dès 1979. Ces premières tentatives d'évaluation ont mis en avant les innovations rendues possibles par la déconcentration des moyens financiers ainsi que les effets positifs des opérations sur l'image de certains quartiers stigmatisés, tout en reconnaissant l'impossibilité de mesurer leur impact sur la vie sociale des territoires visés (Figeat, 1981).

Le lien entre évaluation et politique de la ville s'est affermi avec le passage de la procédure Habitat et vie sociale à la politique de développement social des quartiers (DSQ). Présentés comme des laboratoires où devaient s'expérimenter de nouveaux modes de gestion urbaine, les 148 quartiers visés par un DSQ à partir de 1984 ont vu se multiplier les expériences évaluatives. Ces évaluations locales ont été prolongées par une évaluation nationale, conduite au sein d'un groupe du Commissariat Général du Plan présidé par François Levy. La politique de la ville a alors fourni à cette administration de mission un terrain d'application pour les réflexions méthodologiques sur l'évaluation qu'elle avait développées au cours des années précédentes (cf. Deleau et al., 1986). Fondé sur 16 monographies —dont 4 portaient sur des «sites témoins » qui n'avaient pas bénéficié du DSQ-, le rapport Levy s'ouvre par un chapitre intitulé « DSQ : une politique qui, d'entrée de jeu, appelle l'évaluation », dans lequel le développement social des quartiers est érigé en "terrain privilégié de l'évaluation », du fait «du caractère à la fois global et territorialisé des actions entreprises, (...) de leur aspect exemplaire et des enseignements attendus de leurs résultats, (...) de la décentralisation, qui implique des modes de contrôle des politiques conjointes de l'Etat et des collectivités locales, respectueux de l'autonomie locale et déterminés en fonction d'objectifs plus que de normes étatiques pré-établies » (Levy, 1988). Le rapport d'évaluation, dans lequel le caractère exemplaire du DSQ est réaffirmé à de nombreuses reprises, se concentre sur les conditions de mise en œuvre locale des opérations et leurs réalisations, en laissant dans l'ombre la question de leurs effets et de leur impact. Cette approche conduit à un jugement très positif de la politique menée, insistant sur le caractère novateur et l'apport de la logique expérimentale, tant sur le registre opérationnel (réalisations) que sur celui des modes opératoires (procédure partenariale). La légitimation ainsi apportée à une politique bricolée aux marges des appareils administratifs (Donzelot, Estèbe, 1994) a ouvert la voie à sa pérennisation dans les années 1990. On ne peut cependant conclure au succès de la rétroaction évaluative : si le rapport du Plan a effectivement validé la logique expérimentale du DSQ, il l'a fait sous condition, en soulignant que sa fertilité et sa productivité auraient été menacées par une extension vers un

\footnotetext{
${ }^{5}$ On reprend ici des éléments développés plus longuement dans Epstein, 2007.
} 
grand nombre de sites. Cette condition n'a visiblement pas été entendue, la poursuite du Développement social des quartiers au cours du Xème Plan (1989-1993) s'accompagnant d'un triplement du nombre de quartiers bénéficiant de cette politique.

L'expansion territoriale qui s'est ainsi opérée a marqué l'amorce d'un processus d'institutionnalisation d'une politique érigée au rang de priorité nationale en 1990 par François Mitterrand. Peu de temps après avoir été nommé ministre d'Etat, chargé de la Politique de la ville, Michel Delebarre a installé un Comité national d'évaluation chargé, sous la présidence de Jean-Michel Belorgey, «d'apprécier le contenu, les conditions de mise en œuvre et les effets de cette politique, au regard des objectifs qu'elle poursuit ». Cette évaluation interministérielle a donné lieu à la mise en place d'un lourd dispositif, supposé à la fois garantir sa scientificité et créer les conditions de l'appropriation et de la diffusion de ses résultats ${ }^{6}$.

Disposant d'importants moyens d'études, le Comité a commandé une trentaine de recherches à des équipes universitaires et des bureaux d'études. Cette vague de commandes a ouvert la voie à d'importants progrès sur le plan de la connaissance des quartiers de grands ensembles visés par la politique de la ville, des processus à l'origine de leurs difficultés et surtout des modalités de l'intervention publique en leur direction. L'évaluation des politiques publiques n'est pas cependant qu'une activité cognitive, mais aussi normative et instrumentale (Conseil scientifique de l'évaluation, 1996). Sur ces deux derniers plans, l'évaluation nationale s'est avérée infructueuse ${ }^{7}$. Les membres du comité n'étant jamais parvenus à s'accorder sur les finalités mêmes de la politique de la ville, ils n'ont pu former un jugement partagé sur celle-ci. L'évaluation n'a d'ailleurs pas débouché sur un simple rapport, mais sur deux publications concurrentes. Tout d'abord, à l'approche des élections législatives de 1993, le président du Comité a rédigé en toute hâte un rapport d'évaluation, mais celui-ci n'a pas été validé par le Comité, comme le rappelle son auteur en préambule: le rapport «a été établi au vu des premiers travaux ou prétravaux d'évaluation commandités par le Comité qui lui sont, à la date d'aujourd'hui, parvenus et des diverses prises de position, réflexions et rapports mis en circulation dans le passé récent au sujet de la politique de la ville (...) Les opinions énoncées ont été testées auprès du groupe scientifique du comité d'évaluation, dont les réactions ont été largement prises en compte, mais elles n'engagent que le signataire » (Belorgey, 1993). S'y est ajouté l'Etat animateur, essai publié peu de temps après par les rapporteurs du Comité d'évaluation (Donzelot, Estèbe, 1994). L'existence de deux textes tirant des conclusions fort éloignées des mêmes travaux prouve l'échec du pari évaluatif, consistant dans la production d'un jugement collectif sur la politique considérée dans un premier temps, pour organiser une rétroaction sur celle-ci dans un second temps. L'impact de l'évaluation nationale sur la politique de la ville fut d'autant plus réduit que cette démarche initiée et pilotée par des élus socialistes s'est conclue au moment de l'alternance gouvernementale de 1993, ce qui n'a pas contribué favorablement à la réception politique des travaux du Comité et des propositions formulées par son Président.

On n'en trouve d'ailleurs aucune trace dans le mandat de négociation adressé aux préfets en 1993 pour l'élaboration des contrats de ville du XIe Plan, qui ont pris la suite des conventions de DSQ. Les responsables nationaux de la politique de la ville semblent néanmoins avoir, d'une certaine façon, tiré les leçons de l'échec de l'évaluation nationale, en confiant aux signataires locaux des contrats de ville la responsabilité de l'évaluation. L'incitation nationale à l'évaluation locale s'est appuyée sur une enveloppe financière conséquente (un droit de tirage sur les crédits contractualisés par l'Etat dans les contrats de plan, dont 6/10 000èmes étaient réservés à l'évaluation) et sur la diffusion d'un Dossier-ressources pour l'évaluation des contrats de ville. Reprenant les recommandations du Conseil scientifique de l'évaluation, ce guide méthodologique établi par la Délégation interministérielle à la ville (DIV) insistait sur la nécessité d'un travail préalable de clarification des objectifs, au-delà de ce qui avait été fait dans la phase de négociation locale des contrats. Les conventions établies localement avaient -à de rares exceptions près-

\footnotetext{
${ }^{6}$ Le Comité national d'évaluation, créé sous l'égide du Conseil National des Villes (instance de concertation et de proposition rattachée au Premier ministre), réunissait des élus, des chercheurs, des hauts fonctionnaires et des représentants de la société civile. S'y est ajouté un Groupe inter-administratif d'évaluation chargé de faciliter les relations avec les services de l'Etat, ainsi qu'un Groupe scientifique d'animation réunissant dans un séminaire régulier tous les experts travaillant pour le Comité.
}

${ }^{7}$ Pour une analyse détaillée de l'expérience du Comité national d'évaluation de la politique de la ville, cf. Linhart, 1996 
laissé dans le flou la stratégie retenue pour lutter contre les processus d'exclusion sociale et spatiale, se contentant d'un rappel des grands objectifs de la politique de la ville et des objectifs spécifiques des différents dispositifs qui la composent. L'évaluation est dès lors apparue comme un levier pour mobiliser les acteurs locaux dans un débat sur les hypothèses et objectifs implicites en fonction desquels s'organisait la participation de chacun à un projet collectif, ce qui devait conditionner en retour la pertinence des questionnements évaluatifs et des indicateurs mobilisés pour y répondre. Pour faire émerger ce débat, le dossier-ressources incitait les acteurs locaux à considérer la politique de la ville comme la combinaison de « trois lignes stratégiques à la fois clairement distinctes et rarement isolables en tant que telles, puisque la plupart du temps mêlées dans les politiques locales : la première ligne se donne pour objectif la résorption de poches d'exclusion. Elle vise à réduire les handicaps liés à une concentration spatiale excessive de " cas difficiles». Elle prend appui sur des politiques spécifiques, adaptées, dans une logique de discrimination positive, pour rétablir la norme de l'égalité sociale et de l'homogénéité spatiale. La seconde ligne stratégique vise à prendre en compte l'existence de quartiers populaires. Elle cherche à valoriser les initiatives et les solidarités de voisinage qui permettent de dépasser les différences sociales et de la société locale. Pour ce faire, elle demande à l'action publique de soutenir les initiatives sociales, de favoriser la multiplication des innovations et des expérimentations locales. La dernière ligne stratégique cherche avant tout à restaurer les liens sociaux et urbains qui fondent la cohésion urbaine. Elle insiste moins sur la réduction ou la valorisation de la marge que sur la recomposition de la norme : elle interpelle les données du fonctionnement urbain qui sont à l'origine des processus d'exclusion socio-spatiale et s'attache principalement à la reformulation des politiques publiques sectorielles par une approche nouvelle de la question sociale. » (Délégation interministérielle à la ville, 1995)

Cette grille demeure éclairante pour qui veut suivre les débats actuels relatifs aux enjeux de la politique de la ville et aux interventions publiques développées pour les traiter. La complexité de cette politique et l'instabilité de ses objectifs, qui font l'objet de critiques récurrentes, s'expliquent d'ailleurs largement par la coexistence en son sein de ces trois lignes stratégiques concurrentes. La grille ainsi proposée n'a pourtant pas été d'une grande utilité pour les instances de pilotage des contrats de ville du Xlème Plan et pour les prestataires auxquels ont été confiées les évaluations localement. Parmi la centaine de rapports d'évaluation établis entre 1996 et 1998, ceux qui se sont appuyés sur cette grille pour opérer un travail de clarification des intentions locales -c'est-à-dire une modélisation des liens de causalité supposés entre les données du problème à résoudre, les objectifs visés par les responsables politiques, les réalisations prévues, les effets et l'impact qui en sont attendus- se comptent sur les doigts d'une main'.

Le renvoi de l'évaluation vers le local opéré au cours du Xlème Plan s'est donc conclu par un succès quantitatif (une centaine de contrats de ville ont fait l'objet d'une évaluation in itinere ou ex-post), contrebalancé par un bilan nettement plus mitigé sur le plan qualitatif. A défaut d'avoir suscité des évaluations dignes de cette appellation, l'incitation nationale à l'évaluation locale a contribué à l'émergence d'un marché de l'évaluation, sur lequel sont entrés de nouveaux intervenants. Les chercheurs en sciences sociales, en première ligne dans la conduite des enquêtes évaluatives jusqu'au début des années 1990, ont été remplacés par des bureaux d'études et des cabinets d'audit plus habitués à apporter des réponses ou des solutions à leurs commanditaires qu'à reformuler les questions posées par ces derniers en les accompagnant dans un travail réflexif sur leurs intentions. Ceci explique pour partie le fait qu'une grande majorité des évaluations des contrats de ville a été conduite sans travail préalable de mise en débat des intentions de leurs signataires. La méthodologie unifiée promue par la DIV a alors laissé place à une infinie fragmentation des dispositifs et des approches. La plupart des évaluations se sont réduites à des audits organisationnels, traitant exclusivement du volet institutionnel de la politique de la ville (organisation des dispositifs, fonctionnement des instances, relations entre les « partenaires »...) sans interroger ses finalités et ses réalisations. Lorsque des travaux ont porté sur ses actions, c'est au prix d'une occultation des objectifs généraux de la politique de la ville et de ses modes d'action, qui a singulièrement réduit la portée des analyses. Enfin, les interrogations des maîtrises d'ouvrage locales relatives à l'impact du contrat de ville ont suscité des enquêtes nombreuses et diversifiées (tableaux de bord d'indicateurs statistiques,

\footnotetext{
${ }^{8}$ Pour une analyse approfondie de ces rapports d'évaluation, cf Kirszbaum, 1998 ; Estèbe, Epstein, 1998.
} 
sondages, panels d'habitants, groupes témoins...), mais celles-ci n'ont jamais apporté de réponses concluantes aux questions posées. Car les évaluateurs ont butté sur la difficulté d'identifier ce qui, dans les évolutions observées, pouvait être imputé en propre à une politique additionnelle dont les interventions viennent s'ajouter à celles développées par les différentes politiques sectorielles dans les mêmes territoires. Les évaluations n'ont donc qu'exceptionnellement abouti à des jugements argumentés sur la valeur de la politique menée. Symptomatiquement, de nombreux rapports se concluent par une critique du flou des objectifs poursuivis, lequel limiterait l'évaluabilité de la politique de la ville. . On trouve bien, dans certains rapports d'évaluation, des jugements relatifs à la pertinence, à la cohérence, à l'efficacité et à l'impact du contrat de ville. Mais ces avis aussi tranchés que faiblement étayés ne reflètent que le point de vue de leurs rédacteurs (des bureaux d'études dont l'expertise apparaît très discutable) et non le résultat de la mise en débat de leurs enquêtes. Quant à l'exigence de publicité des rapports d'évaluation, elle semble avoir été systématiquement oubliée...

La mise en place d'une nouvelle génération de contrats de ville en 2000 s'est néanmoins accompagnée de la réaffirmation d'une forte ambition en matière d'évaluation, présentée comme « un impératif d'efficacité et une exigence démocratique $\aleph^{10}$. Un Comité national d'évaluation de la politique de la ville a été installé en janvier 2002 et un nouveau guide méthodologique de l'évaluation des contrats de ville a été diffusé par la DIV quelques mois plus tard. La déconvenue a été à la hauteur de l'ambition affichée. Le Comité national ne s'est réuni qu'à deux reprises, et il n'a eu aucune production propre. S'agissant des évaluations locales, le bilan est comparable à celui du Xle Plan. A mi-parcours, plus de la moitié des contrats de ville 20002006 avaient fait l'objet d'une évaluation. Mais ces évaluations locales reproduisaient les travers méthodologiques (et déontologiques) observés quelques années plus tôt, quand elles ne les amplifiaient pas (Epstein, Kirszbaum, 2005).

Quelques mois après avoir été nommé ministre délégué à la Ville et à la Rénovation urbaine dans le premier gouvernement de Jean-Pierre Raffarin, Jean-Louis Borloo a demandé à ses services d'établir une synthèse de ces évaluations à mi-parcours des contrats de ville 2000-2006. Après avoir réuni les rapports disponibles et tenté de les exploiter, la délégation interministérielle à la ville a dû reconnaître qu'elle était dans l'incapacité à répondre à la demande ministérielle. A son corps défendant, elle a ainsi validé l'analyse développée par les magistrats de la Cour des comptes dans le rapport particulier sur la politique de la ville qu'ils avaient publié en 2002: cette politique serait marquée par des tares conceptuelles qui empêcheraient son évaluation. Pour la rendre possible, les hauts magistrats rappelaient les demandes qu'ils avaient déjà formulées dans leur rapport annuel en 1995 : clarification des objectifs de la politique de la ville; déclinaison de ces objectifs dans des indicateurs de résultats; identification plus précise des programmes y contribuant, ainsi que des ressources budgétaires et extra-budgétaires qui leur sont consacrés; amélioration des systèmes statistiques d'observation des quartiers. Autant de demandes auxquelles la loi Borloo a répondu.

Avant de considérer cette réforme de la politique de la ville qui semblait annoncer un renouveau de son évaluation, tirons un bilan d'ensemble des expériences antérieures. Plus que toute autre politique publique, la politique de la ville a affiché de fortes ambitions en la matière, sans toutefois aller jusqu'à engager de méta-évaluation (évaluation des évaluations) dont les résultats auraient probablement été cruels. Combler ce manque supposerait d'engager des démarches spécifiques. On peut néanmoins pointer l'écart qui

\footnotetext{
${ }^{9}$ Ce faisant, les chargés d'évaluation ont renvoyé la responsabilité de l'échec de la démarche sur leurs commanditaires. Cette forme de mise en cause n'est pas propre à l'évaluation de la politique de la ville. Elle se retrouve fréquemment dans les discours et les écrits des professionnels français de l'évaluation, qui se singularisent par une surprenante propension à l'autocélébration et, conjointement, à l'incrimination des responsables politiques pour lesquels ils travaillent, dont ils stigmatisent le manque d'intérêt voire la frilosité à l'égard de l'évaluation (cf. Monnier, Henard, 2001). Cette analyse n'est pas dénuée de fondements, mais elle apparaît un peu courte. S'agissant de la politique de la ville, les évaluations ont été le plus souvent externalisées, confiées globalement à des bureaux d'études chargés à la fois de la conception des dispositifs, de leur animation, de la production de données et de la rédaction du rapport final. Les évaluateurs sont donc, a minima, coresponsables du succès comme de l'échec des démarches entreprises.

${ }^{10}$ Circulaire du ministre délégué à la Ville du 13 novembre 2000 relative à l'évaluation des contrats de ville et des politiques régionales de la ville pour la période 2000-2006.
} 
sépare les évaluations de la politique de la ville de l'idéal évaluatif. A l'aune des critères méta-évaluatifs utilisés pour apprécier la qualité des évaluations -utilité ; exactitude et caractère systémique des travaux; compétence et intégrité des évaluateurs; publicité accordée aux résultats, à la fois positifs et négatifs (Stufflebeam, 2001)-, la quasi-totalité des évaluations qui ont pris pour objet la politique de la ville au cours du dernier quart de siècle doivent être considérées comme des échecs. Aucune de ces démarches évaluatives n'est parvenue à répondre de façon satisfaisante aux principales questions qui ont justifié leur lancement ${ }^{11}$, et les nombreuses réorientations qu'a connues cette politique ne se sont jamais appuyées sur les recommandations issues des évaluations (Jacquier, 2004). A défaut de réaliser son propre programme, l'évaluation a néanmoins été utile pour la politique de la ville, assurant des fonctions diverses au fil de son histoire. Elle a tout d'abord légitimé cette politique expérimentale portée par des acteurs marginaux dans le système politico-administratif, contribuant ainsi à sa pérennisation. Elle a ensuite servi de cadre à la théorisation de la politique de la ville, fournissant à ses porteurs nationaux et locaux des ressources cognitives et/ou discursives mobilisables vis-à-vis de leurs partenaires pour les inciter à s'engager dans une politique dont ils se tenaient souvent à l'écart. Enfin, le renvoi de l'évaluation vers le local a abouti, dans de nombreuses villes, à la constitution d'outils de suivi et d'observation qui ont pallié les carences d'un appareil statistique national structuré pour répondre aux besoins de connaissances de l'Etat et non à ceux des pouvoirs locaux (Desrosieres, 1993). De manière plus exceptionnelle, l'évaluation a fait émerger des scènes de débat local sur la stratégie poursuivie, les réalisations, les effets de la politique de la ville, qui ont permis d'infléchir la politique menée ${ }^{12}$.

\section{Le programme national de rénovation urbaine sera-t-il évalué ?}

L'approche transversale, partenariale et expérimentale qui a guidé la politique de la ville de la fin des années 1970 au début des années 2000 a justifié l'investissement de ses acteurs dans l'évaluation, en même temps qu'elle peut expliquer les difficultés rencontrées par ces derniers pour faire fructifier cet investissement. La loi d'orientation et de programmation pour la politique de la ville et la rénovation urbaine du $1^{\text {er }}$ août 2003 marque la fin de cette approche. Elle s'apparente à une opération de design institutionnel (Schneider, Ingram, 1997) redéfinissant le problème traité, les cibles visées, les objectifs effectivement poursuivis ainsi que la distribution du pouvoir et des responsabilités au sein du système d'acteurs en charge de sa mise en œuvre (Epstein, 2005). Cette évolution ne s'appuie en rien sur les évaluations passées. Au contraire, l'échec évaluatif a été mis en avant pour justifier la réforme opérée, dont les lignes directrices avaient été dessinées dans le rapport de la Cour des comptes de 2002.

Tout, dans la loi Borloo, a été fait pour rendre la politique de la ville évaluable. Les récits des acteurs ayant participé à son élaboration donnent même à voir une inversion des rapports entre la politique de la ville et son évaluation : les outils de l'évaluation n'ont pas été construits en fonction des objectifs impartis à cette politique ; au contraire, ce sont les enjeux et les objectifs de cette politique publique qui ont été redéfinis en fonction de l'exigence de mesure des résultats et des instruments administratifs disponibles pour ce faire. La politique de la ville s'organise désormais autour de programmes pluriannuels dont les objectifs sont définis dans la loi. Formulés en termes de réduction des inégalités sociales et des écarts de développement entre les 751 zones urbaines sensibles (ZUS) et l'ensemble du territoire national, ces objectifs sont déclinés dans une batterie de 65 indicateurs quantitatifs réunis dans l'annexe 1 de la loi. Cette opération de clarification se prolonge sur le plan des moyens. L'incertitude qui prévalait jusque là

\footnotetext{
${ }^{11}$ Notamment celles relatives à l'impact de la politique de la ville ou, plus simplement, à ses effets sur le plan de la discrimination positive territoriale. En la matière, divers rapports administratifs et scientifiques ont souligné la persistance d'importantes inégalités de traitement, au détriment des quartiers pourtant dits « prioritaires » : les ressources supplémentaires qui leur sont consacrées par la politique de la ville et divers programmes connexes (Zones d'Education Prioritaires notamment) ne suffisent pas à compenser l'inéquitable distribution des moyens des politiques de droit commun. Cf. Treguer, 2002 ; Fourcade et al., 2005 ; DARES, 2007 ; Davezies, 1999 ; Benabou et al., 2004.

${ }^{12}$ C'est notamment le cas de l'évaluation régionale conduite à la fin des années 1990 en lle-de-France, qui a abouti à la définition d'une grille de lecture régionale des processus d'exclusion et à des propositions d'adaptation des politiques régionales à ces différents types de territoires (Bravo, 1999). Mais il s'agit là d'une exception qui, par contraste, met en lumière la distance qui sépare les pratiques développées ailleurs de la théorie évaluative.
} 
quant aux ressources dévolues à la politique de la ville laisse place à un système bien plus lisible, avec la création d'une Agence nationale pour la rénovation urbaine (ANRU) mutualisant l'ensemble des apports financiers de l'Etat, du 1\% logement, de la Caisse des dépots et du mouvement HLM. Enfin, un Observatoire national des zones urbaines sensibles (ONZUS) est créé, chargé de « mesurer l'évolution des inégalités sociales et des écarts de développement dans chacune des zones urbaines sensibles, suivre la mise en œuvre des politiques publiques conduites en leur faveur, mesurer les moyens spécifiques mis en œuvre et en évaluer les effets par rapport aux objectifs et aux indicateurs de résultats » définis par le législateur. Prolongeant ce souci évaluatif, la loi impose aux communes et communautés d'agglomération l'établissement d'un « bilan annuel des actions menées dans les ZUS de leur territoire, des moyens qui y sont affectés et de l'évolution des indicateurs relatifs aux inégalités ", lequel doit être présenté à l'occasion du débat sur les orientations générales de leur budget.

L'édifice évaluatif a été parachevé un an plus tard, avec l'institution d'un Comité d'évaluation et de suivi au sein de l'ANRU chargé, suivant les termes du décret qui lui a donné naissance, "d'apprécier la mise en œuvre du programme national de rénovation urbaine en rendant des avis chaque fois qu'il le juge utile ou à la demande du président du conseil d'administration ». Deux des membres dudit comité ont par la suite rejoint le gouvernement ${ }^{13}$, ce qui semblait créer les conditions d'une rétroaction évaluative. Six ans après le vote de la loi Borloo, dont les programmes devaient prendre fin en $2008^{14}$, les promesses évaluatives ontelles été tenues?

On notera tout d'abord que les travaux de l'ONZUS ont permis des avancées substantielles sur le plan de l'observation. Ses rapports annuels ont apporté des informations statistiques inédites sur les ZUS, ainsi que sur les ressources qui leur sont dévolues. II en ressort notamment que la politique de la ville n'est pas parvenue à améliorer la situation socio-économique de ces quartiers, où réside $8 \%$ de la population française. Au contraire, la dynamique d'accroissement des écarts territoriaux s'est prolongée sans discontinuer, sous l'effet d'une concentration croissante des populations peu qualifiées, pauvres, précaires et issues de l'immigration. Le seul impact sensible de la politique de la ville relevé par l'ONZUS concerne la dotation en équipements publics, qui sont aussi voire plus nombreux dans les ZUS que dans les autres quartiers. Mais cette présence n'est souvent pas à hauteur des besoins locaux, bien plus importants qu'ailleurs. Dans l'ensemble, l'implantation d'équipements collectifs dans les quartiers prioritaires ne s'est pas prolongée par une réduction des inégalités d'accès à l'offre publique, y compris sur les thématiques privilégiées par la politique de la ville : les pratiques sportives, culturelles et de loisirs des habitants des ZUS demeurent nettement plus limitées que celles de l'ensemble des citadins, y compris pour des activités socialement et culturellement peu marquées. Ce constat d'échec de la politique de la ville doit cependant être nuancé. Comme le rappellent chaque année les rédacteurs du rapport de l'ONZUS, les statistiques qui y sont réunies portent sur la situation moyenne des 751 ZUS françaises, marquées par des situations et des dynamiques hétérogènes. L'échec global masque donc des succès locaux. II n'en demeure pas moins que l'objectif national de réduction des écarts défini par le législateur en 2003 n'a pas été atteint, ce qui s'explique en grande partie par l'importance des mobilités résidentielles des habitants des ZUS : l'analyse détaillée des profils des entrants et des sortants établie par l'ONZUS en 2005 montre que les populations les plus fragiles ont tendance à rester dans ces quartiers alors que les trajectoires sociales ascendantes s'accompagnent souvent d'un déménagement hors des ZUS. Selon la date d'observation, ce ne sont donc pas forcément les mêmes habitants qui y résident. La dégradation des indicateurs socio-économiques de ces quartiers résulte donc avant tout des changements de population induits par la mobilité résidentielle, et ne renseigne pas nécessairement sur l'évolution des conditions de vie des populations présentes en début de période.

L'amélioration des systèmes d'observation à laquelle s'est attelé l'ONZUS, en lien avec l'ensemble des organismes de la statistique publique, a indéniablement fait progresser la connaissance des quartiers visés par la politique de la ville et de leurs dynamiques, ainsi que des moyens mobilisés par cette politique. Les

\footnotetext{
${ }^{13}$ Fadela Amara, nommée secrétaire d'État chargée de la Politique de la ville en 2007, suivie par le président du Comité, Yazid Sabeg, devenu Commissaire à la diversité et à l'égalité des chances en 2008

${ }^{14}$ Plusieurs lois ont prolongé le Programme national de rénovation urbaine jusqu'en 2013.
} 
progrès de l'observation ont ainsi ouvert la voie à des avancées substantielles de l'évaluation nationale de la politique de la ville, en rendant possible, par des analyses économétriques, la mesure de l'efficacité de ses différents programmes ${ }^{15}$. Si l'on adopte une conception technocratique de l'évaluation, considérant que l'exercice vise justement à reconnaître les effets propres d'une politique publique (Deleau et al., 1986), il convient de souligner la cohérence et le succès de la réforme opérée en 2003. En revanche, si l'on privilégie une conception plus démocratique de l'évaluation, lui conférant la charge « d'accroître la qualité de la vie démocratique » en formant un jugement collectif sur la valeur d'une politique publique afin de nourrir le débat public (Viveret, 1989), il faut convenir que les avancées sont bien maigres, comme l'illustre le programme national de rénovation urbaine (PNRU), pièce maîtresse de la loi Borloo.

Alors que des ressources considérables sont mobilisées pour conduire les opérations de rénovation urbaine, sans commune mesure avec celles dévolues aux autres procédures développées par la politique de la ville en direction des mêmes quartiers, ces opérations sont les seules pour lesquelles le niveau national n'exige pas la mise en place de dispositifs d'évaluation locale. Un an après le terme initialement prévu de l'ambitieux programme de rénovation urbaine, dont le coût total est estimé à 42 milliards d'euros, le titre de l'article publié en 2005 par un membre du Comité d'évaluation et de suivi de l'ANRU demeure d'actualité : «L'ANRU, au risque de l'évaluation: Que va-t-on réellement évaluer et quand va-t-on s'y mettre ?» (Robert, 2005). On pourrait y ajouter une question supplémentaire : qui doit évaluer le PNRU ? La répartition des rôles entre l'ONZUS et le Comité d'évaluation et de suivi de l'ANRU demeure incertaine. L'ONZUS est une structure d'observation, portée par la DIV ${ }^{16}$, et non une instance évaluation. Ses rapports fournissent une matière indispensable à l'évaluation, mais ses responsables ont jusqu'à présent fait preuve d'une grande prudence, s'interdisant de formuler tout jugement sur la valeur du PNRU. II en va différemment du Comité d'évaluation et de suivi de l'ANRU, qui a multiplié les critiques sur la mise en œuvre du programme. Mais ce comité n'est pas véritablement parvenu à s'imposer comme l'instance d'évaluation du PNRU. Faute de mandat formel définissant son rôle et en l'absence de démarches préliminaires qui lui auraient permis de se doter d'un référentiel d'évaluation structurant ses travaux dans la durée, le comité s'est positionné dans un rôle de commentateur chemin faisant de la mise en œuvre plutôt que d'évaluateur: les hypothèses fondatrices de la rénovation urbaine, relatives aux effets sociaux de la mixité résidentielle et de la banalisation urbaine, ne sont jamais discutées dans ses rapports, qui se concentrent sur les moyens dévolus à la rénovation urbaine, ainsi que sur les conditions de pilotage du programme national et des opérations locales.

A décharge du comité, il faut souligner que celui-ci s'est heurté à l'absence d'informations cruciales, nécessaires pour juger de l'effectivité du programme et, a fortiori, pour juger de son efficacité. Les systèmes de suivi mis en place par l'ANRU ont été structurés dans une perspective de gestion financière, et non d'évaluation. S'ils rendent compte de l'avancement des opérations locales, c'est de façon très imparfaite, puisqu'ils ne permettent pas de distinguer précisément les opérations achevées, en cours et en préparation. Ne disposant pas de sources de données administratives fiables lui permettant de juger de la performance du PNRU -i.e. de son efficacité socio-économique, de ses effets sur la qualité de vie dans les quartiers ou de l'efficience de la gestion de l'ANRU-, le comité d'évaluation s'est contenté d'informations disparates et parcellaires réunies par le biais de visites de terrain, d'auditions et d'études monographiques. II a fallu attendre le début de l'année 2009 pour qu'il engage un programme d'études conséquent, susceptible d'aboutir à la constitution d'une vision plus générale de la mise en œuvre du PNRU et de ses effets. Si les bases évaluatives ont ainsi été -tardivement- posées, on peut craindre que l'édifice ne sorte jamais de terre: un amendement au projet de loi de finances pour 2010 voté par la commission des finances du Sénat a supprimé les crédits du comité d'évaluation et de suivi ${ }^{17}$. A la date de rédaction de ces

\footnotetext{
${ }^{15}$ Les bases théoriques et les méthodes de l'évaluation économétrique des politiques de développement social sont présentées, exemples à l'appui, sur le site du Poverty Action Lab (www.povertyactionlab.com). Pour une première application au programme des Zones Franches Urbaines cf. Rathelot, Sillard, 2007.

${ }^{16}$ Renommée Secrétariat général du Comité interministériel des villes en 2009

${ }^{17}$ Suivant la présentation qui en est faite par son auteur, le Sénateur UMP de Seine-Saint-Denis Philippe Dallier, cet amendement a « pour objet d'ouvrir une réflexion sur la suppression du comité de suivi et d'évaluation de l'ANRU dont les frais de fonctionnement courant - hors titre 2- ont dépassé le montant de 300.000 euros malgré une activité réduite depuis 2008. Ce
} 
lignes, on ne sait si cet amendement sénatorial survivra à la suite de la procédure d'examen du budget ; son adoption par la commission des finances constitue néanmoins un rappel à l'ordre en direction du comité, de bien mauvais augure pour la suite de ses travaux et, plus largement, pour l'avenir de l'évaluation en France.

L'absence des éléments nécessaires à la mise en débat public du PNRU est longtemps passée inaperçue, occultée par une politique de communication intense de l'ANRU, qui a diffusé un flux continu de chiffres et d'images au fort pouvoir évocateur largement repris par la presse. L'état d'avancement du PNRU rendu public mensuellement par l'agence a ainsi donné à voir une croissance continue du « chantier du siècle », rendu tangible par les images spectaculaires des explosions de tours et de barres. Mais cet état d'avancement ne fournissait aucune indication sur les réalisations effectives, renseignant simplement sur les réalisations programmées, qui étaient systématiquement rapportées aux objectifs quantitatifs arrêtés dans la loi Borloo. Au cours des quatre ans qui ont suivi le vote de cette loi, nul n'a su combien de logements avaient été démolis ou reconstruits. II a fallu attendre la fin de l'année 2007 pour que des premiers éléments chiffrés soient rendus publics par l'ONZUS : à mi-parcours du PNRU, les opérations engagées s'élevaient tout juste à $10 \%$ des objectifs définis par le législateur. A la fin 2006 , la construction de 19800 logements avait été engagée, pour 28500 démolitions, soit un ratio de 70\% fort éloigné de l'objectif de reconstruction intégrale défini dans la loi. En outre, la typologie des logements reconstruits différait fortement de celle des logements démolis: les grands logements (T5 et plus) représentaient la moitié des démolitions (48\%), mais seulement un dixième des reconstructions. Ces dernières correspondent, dans deux tiers des cas (64\%), à des petits logements (du studio au T3), lesquels ne représentent qu'un quart des démolitions (24\%).

Les informations contenues dans le rapport 2007 de l'ONZUS ont ainsi confirmé les craintes exprimées au cours des années précédentes par quelques observateurs (Fondation Abbé Pierre, 2006), qui n'avaient eu qu'un écho limité : la mise en œuvre du PNRU conduit à une réduction de l'offre de logement social, en particulier sur les segments du parc pour lesquels le déficit est déjà marqué, réduisant ainsi les possibilités de mobilité résidentielle des locataires HLM et plus encore les perspectives d'accès au logement social des mal-logés, en dépit de l'entrée en application d'un droit opposable au logement à la fin de l'année 2008. A cet égard, les premiers effets directs du PNRU apparaissent singulièrement contrastés : si de nombreux ménages déplacés à l'occasion des démolitions-reconstructions expriment leur satisfaction quant à leurs conditions de relogement (Lelévrier, 2008), ces opérations ont aussi pu contribuer à l'approfondissement de la crise du logement dans les territoires marqués par d'importants décalages entre l'offre de logement sociaux et les besoins en la matière. Plus substantiellement, des observations locales parcellaires laissent entrevoir d'importants effets pervers, au regard de l'objectif de rétablissement de la mixité sociale qui structure le PNRU : la mise en œuvre des opérations peut conduire à l'accélération des départs des ménages les moins défavorisés, et au renforcement de micro-concentrations des ménages les plus défavorisés et des familles nombreuses à l'échelle de quelques bâtiments ou sous-quartiers. Autant de signaux qui conduisent à souligner l'urgence d'un débat évaluatif sur les effets et l'impact du PNRU, et plus encore sur l'efficience de ce programme qui absorbe une part croissante des ressources publiques dévolues au logement social. Tout laisse cependant penser qu'il faudra attendre le terme des opérations amorcées dans des centaines de quartiers pour qu'un tel débat puisse s'engager. Loin de faire émerger ce débat, les émeutes de l'automne 2005 en ont éloigné la perspective. La rénovation urbaine a été tenue à l'écart des controverses politiques et des débats académiques qui ont accompagné et prolongé l'embrasement de centaines de quartiers. Au contraire, cet événement traumatique a conduit l'ensemble des responsables politiques à réaffirmer l'urgence de la rénovation urbaine et le primat de l'action sur le temps long de la réflexion et de la rétroaction, affirmation qui s'est prolongée par l'allocation de ressources budgétaires supplémentaires à l'ANRU. Depuis lors, les écarts entre les quartiers prioritaires de la politique de la ville et leur environnement se sont accrus sans discontinuer, sans que cela conduise à une amorce

comité a eu une utilité au moment de la mise en place de l'ANRU. II ne s'impose plus dès lors que les règles du jeu sont désormais clairement établies et que les échanges entre les différents acteurs (porteurs de projets, bailleurs, financeurs, délégués de l'ANRU) sont en réalité la meilleure garantie de leur respect et de la réussite des projets dans l'intérêt des habitants des quartiers et des collectivités concernées ». 
de mise en débat du PNRU, si l'on excepte les polémiques relatives au désengagement de l'Etat de son financement.

Le PNRU s'appuie, comme tout programme d'action publique, sur un jeu d'hypothèses dont la validité est très incertaine, mais qui semble dans le cas présent indiscutable. Si la loi Borloo a créé les conditions de possibilité d'une mesure des effets et de l'impact des programmes de la politique de la ville, ces conditions ne sont pas suffisantes pour que ceux-ci fassent effectivement l'objet d'une évaluation démocratique, au sens du rapport Viveret. On peut d'ailleurs penser que la possibilité technique d'évaluer l'efficacité, l'efficience et l'impact du principal programme de la politique de la ville constitue désormais la raison principale des blocages évaluatifs, qui relèvent du registre politique. Car l'évaluabilité de la politique de la ville créé, par contrecoup, des risques pour les responsables politiques et administratifs qui en ont la charge. La mise en place du PNRU ayant été accompagnée d'opérations de communication politique d'une redoutable efficacité, au bénéfice des responsables de ce programme, ces derniers ont de bonnes raisons de ne pas se hâter dans la réalisation d'évaluations dont tout laisse penser qu'elles révéleront un jour l'immensité des écarts entre les résultats attendus, les résultats précocement annoncés et ceux qui seront effectivement mesurés au terme du programme.

\section{Des politiques publiques aux programmes : l'évaluation sauvée par la LOLF ?}

Votée à l'initiative du Parlement le 1er août 2001, la loi organique relative aux lois de finances est fréquemment présentée comme une «révolution budgétaire » dotant la France d'une «nouvelle constitution financière ». Succédant à une ordonnance de 1959 qui a régi pendant près d'un demi-siècle le droit budgétaire français, ce texte organique a introduit une nouvelle présentation du budget en loi de finances. Les charges budgétaires de l'Etat sont désormais regroupées par mission, chacune comprenant un ensemble de programmes réunissant «les crédits destinés à mettre en œuvre une action ou un ensemble cohérent d'actions relevant d'un même ministère et auquel sont associés des objectifs précis, définis en fonction de finalités d'intérêt général, ainsi que des résultats attendus et faisant l'objet d'une évaluation» (article 7). Les annexes explicatives jointes au projet de loi de finances comprennent les projets annuels de performance de chaque programme, qui présentent de façon extrêmement détaillée les finalités et le contenu de l'action publique conduite et financée par l'Etat : stratégie, objectifs et indicateurs de performance associés. Chaque responsable de programme (un haut fonctionnaire désigné par le ministre compétent pour piloter un programme donné) s'engage ainsi devant le Parlement, à l'occasion de l'examen de la loi de finances, sur des objectifs chiffrés pour l'année suivante. II doit ensuite rendre compte des résultats obtenus et expliquer les écarts par rapport aux prévisions dans un rapport annuel de performances, joint au projet de loi de règlement rendant compte de l'exécution budgétaire de l'année passée.

Les réformateurs de Bercy insistent sur l'importance du volet performance de la loi organique. Celui-ci a vocation à introduire une plus grande transparence dans l'action de l'Etat et à améliorer le pilotage de ses politiques, puisque les objectifs et indicateurs de performance sont destinés à être déclinés tout au long des organisations administratives (Atkwright et al., 2007). Les améliorations de la gestion publique attendues de cette réforme budgétaire relèvent donc à la fois du registre démocratique et gestionnaire. Sur le plan démocratique, la nouvelle procédure budgétaire doit non seulement accroître la transparence et l'accountability, mais aussi restituer au Parlement une capacité d'arbitrage et de définition des programmes de l'Etat qu'il avait largement perdu (Siné, 2006). Sur le plan de l'efficience gestionnaire, des progrès sont attendus de l'introduction d'une culture managériale dans des administrations dont les agents n'étaient jusque-là pas responsables des résultats de leur action, dès lors qu'ils utilisaient les moyens conformément aux normes juridiques. Le changement concerne en premier chef les responsables de programme, qui bénéficient d'une plus grande autonomie dans la gestion de leurs moyens pour atteindre les objectifs votés par le Parlement. La globalisation et la fongibilité des crédits confèrent à ces managers une grande liberté 
pour redéployer les crédits au sein du programme dont ils sont responsables ${ }^{18}$. Cette liberté de gestion est la contrepartie d'un engagement sur des objectifs assortis d'indicateurs mesurant les résultats des actions menées, dont les responsables doivent rendre compte à leur ministre et au Parlement.

En clarifiant les objectifs poursuivis, en identifiant les moyens mobilisés et en mesurant les résultats obtenus, la nouvelle procédure budgétaire rend possible une réelle évaluation de l'efficacité et de l'efficience des programmes de l'Etat, en rupture avec une évaluation des politiques publiques trop souvent centrée sur les seule procédures formelles. Entrée en vigueur par étapes, la LOLF s'est pleinement appliquée à compter de la loi de finances pour 2006. Au terme des deux premiers exercices de ce nouveau régime budgétaire, force est de reconnaître que les promesses évaluatives de la loi organique restent à concrétiser.

A l'heure actuelle, les objectifs et les indicateurs définis dans les projets annuels de performance « forment un ensemble à la fois pléthorique et carencé » (Maury , 2008), qui ne peut servir de base à une évaluation systématique des programmes de l'Etat : le projet de loi de finances pour 2007 comprenait 634 objectifs et 1295 indicateurs pour l'ensemble du budget! Les valeurs-cibles associées à nombre de ces objectifs apparaissent totalement irréalistes, relevant plus directement de l'affichage d'un volontarisme politique que d'un souci pragmatique d'amélioration de la performance de l'action publique. A cela s'ajoutent les très nombreuses imperfections du système d'indicateurs mis en place: instabilité, non-disponibilité des données nécessaires pour les renseigner et surtout pertinence singulièrement limitée. Car ces indicateurs dits « de performance » informent souvent exclusivement sur l'activité et les moyens des administrations, ou fournissent un éclairage en trompe l'œil sur les résultats de leur action. C'est notamment le cas lorsque leur évolution ne peut être directement imputée à un programme étatique donné, parce que dépendant principalement ou partiellement du contexte, d'autres programmes ou d'autres acteurs.

On peut penser qu'il s'agit là de faiblesses temporaires, appelées à s'effacer avec le temps (Arthuis, 2005 ; Maury, 2008). On peut aussi penser qu'elles sont appelées à perdurer : près de quatre ans après la remise d'un rapport d'information dans lequel le sénateur Arthuis analysait précisément les limites des objectifs et des indicateurs retenus, leur nombre n'a diminué que de $8 \%$ et leur qualité demeure très inégale. Le cas du PNRU, qui a basculé par anticipation dans le régime LOLF, conduit plus substantiellement à s'interroger sur les limites du système d'objectifs et d'indicateurs mis en place, du point de vue de l'évaluation : et si le problème n'était pas seulement technique, mais aussi politique?

La loi d'orientation et de programmation pour la ville et la rénovation urbaine a été élaborée dans la période d'entrée en application progressive de la LOLF votée deux ans plus tôt. Les principes et les procédures promus par le texte organique pour améliorer la performance et l'évaluabilité des politiques publiques ont inspiré les rédacteurs de la loi Borloo, qui devaient alors faire face à une vague de critiques portant justement sur l'inefficacité de la politique de la ville et son déficit d'évaluation. Si la loi Borloo ne découle pas directement de la loi organique, cette dernière a pesé dans l'élaboration de la première, qui a été façonnée de telle manière qu'elle se conforme, par anticipation, aux exigences de la « nouvelle constitution financière ». L'indéfinition nationale des objectifs de la politique de la ville, qui s'accompagnait d'une grande difficulté pour identifier ses ressources, ont laissé place à des programmes pluriannuels structurés par des objectifs de résultats -auquel sont associés des indicateurs quantitatifs suivis par un observatoire national-, dont la mise en œuvre ne dépend plus des contributions de divers ministères, mais s'appuie sur des lignes parfaitement identifiées dans le budget de l'Etat. Les options organisationnelles retenues pour le PNRU témoignent de ce souci de conformation anticipée à « l'esprit de la LOLF », avec la création d'une agence autonome responsable de la mise en œuvre du PNRU, bénéficiant d'une liberté managériale quasi-totale dans l'utilisation des ressources consacrées à ce programme par l'Etat et ses partenaires nationaux.

\footnotetext{
${ }^{18}$ Cette liberté est limitée par le principe de « fongibilité asymétrique », qui se traduit par la faculté d'utiliser à d'autres emplois les crédits prévisionnels dédiés aux dépenses de personnel, sans que l'inverse puisse être réalisé. Les crédits de personnels sont donc limitatifs par programme.
} 
A bien des égards, la politique de rénovation urbaine correspond au modèle néo-managérial promu par la LOLF : ses objectifs ont été précisément définis par le législateur, dans une loi d'orientation et de programmation ; l'organisation administrative de la rénovation urbaine (ANRU) et l'architecture budgétaire de la LOLF (programme 202) se recoupent parfaitement; les responsabilités de l'agence en charge du programme et de son administration de tutelle (la DIV) sont apparemment clairement distinguées. En dépit de cela, la rénovation urbaine semble toujours résister au régime évaluatif LOLFien, cinq ans après le lancement du PNRU.

Les objectifs et indicateurs qui figurent dans les projets annuels de performance du programme « rénovation urbaine » ne dérogent pas au cas général. Leur instabilité est spectaculaire: des trois objectifs et sept indicateurs réunis dans le projet annuel de performance du programme "rénovation urbaine » en 2006, seuls un objectif et deux indicateurs se retrouvent dans le projet de l'année suivante. Les changements ainsi apportés dans la batterie d'indicateurs limitent les possibilités de suivi pluriannuel, sans lequel l'appréhension des performances et de leur évolution dans le temps ne peut être réellement significative. L'incomplétude des objectifs et indicateurs qui figurent dans les projets annuels de performance de ce programme (et du programme "Equité sociale et territoriale et soutien», qui correspond au volet social de la politique de la ville) est tout aussi criante, puisqu'ils ne couvrent qu'une infime partie de ceux qui figurent dans la loi d'orientation et de programmation pour la politique de la ville et la rénovation urbaine. On peut même parler d'incohérence, en l'absence de liens entre l'objectif général et les indicateurs définis dans la loi et les critères d'appréciation de l'efficacité socio-économique, de la qualité de service et de l'efficience de la gestion qui figurent dans les projets annuels de performance ${ }^{19}$. Dans le cas de la rénovation urbaine, l'ANRU a fortement pesé sur la formulation des objectifs et indicateurs présentés au Parlement, imposant sa conception de la performance. L'appréciation de cette dernière dans le cadre de la procédure budgétaire s'organise donc moins au regard des finalités définies par le législateur ou des objectifs précisés par l'administration qui en a la responsabilité que de la traduction qui en a été donnée par l'opérateur unique du programme. Soit une inversion totale des rapports entre Parlement, administration et agence qui devaient découler du bel ordonnancement de la LOLF.

Plus encore, ce cas nous rappelle le caractère proprement politique de l'évaluation, qui ne peut se réduire aux seules dimensions organisationnelle, instrumentale et managériale. Toutes les conditions d'une évaluation du PNRU sur les registres de l'efficacité, de l'efficience et de l'impact sont réunies, mais ces conditions nécessaires ne sont visiblement pas suffisantes. Les cinq premières années de mise en œuvre de ce programme révèlent en effet des pratiques fort éloignées de celles que les discours des promoteurs de la LOLF pouvaient laisser espérer. Les transformations organisationnelles et procédurales inspirées par la loi organique ont assurément permis aux responsables de la rénovation urbaine de développer une communication performante ; les effets sont plus incertains sur le plan de la transparence et de l'efficience de l'action menée. L'évaluation du PNRU s'est continûment heurtée aux stratégies de protection des responsables politiques et administratifs en charge du programme, qui ont multiplié les initiatives relevant du blame avoidance (Weaver, 1986) pour neutraliser une évaluation susceptible de mettre en doute le succès annoncé du programme. Le cas de la rénovation urbaine prouve donc que les difficultés de l'évaluation des programmes de la LOLF ne sont pas simplement techniques et éphémères, correspondant

\footnotetext{
${ }^{19}$ La loi du $1^{\text {er }}$ août 2003 arrête, dans son article premier, un objectif de réduction des inégalités sociales et des écarts de développement entre les territoires. Le projet annuel de performance 2008 de la mission Rénovation urbaine s'organise pour sa part autour de quatre objectifs déclinés en huit indicateurs : maintenir l'offre de logements sociaux dans le cadre du PNRU (indicateur associé : taux de couverture des démolitions par des reconstructions); améliorer la qualité de l'habitat pour les habitants des quartiers concernés par le PNRU (indicateurs associés : différentiel entre l'indice de satisfaction des habitants des ZUS quant à la qualité de l'environnement de leur logement et celui constaté dans les unités urbaines correspondantes ; nombre de PLUS-CD financés par rapport au nombre de PLUS-CD programmés; différentiel entre le taux de vacance des logements sociaux en ZUS et celui observé dans les unités urbaines correspondantes) ; concentrer la mise en oeuvre du programme de rénovation urbaine sur les secteurs les plus fragiles (indicateur associé : part des crédits mobilisés pour la rénovation urbaine selon le niveau de priorité des zones à rénover) ; maîtriser le coût et les délais du programme national de rénovation urbaine (indicateurs associés : évolution du coût moyen des opérations d'investissement financées dans le cadre du programme national de rénovation urbaine; part des opérations programmées dont l'engagement est retardé; taux d'engagement des programmations annuelles).
} 
à une phase d'apprentissage administratif. Elles sont aussi politiques, renvoyant à des dimensions plus structurelles de l'action publique, notamment aux besoins de légitimation des responsables politiques et administratifs soumis aux exigences de la compétition électorale pour les premiers, de la concurrence entre segments administratifs pour l'accès à des ressources de plus en plus rares pour les seconds.

On pourrait objecter qu'il est difficile de monter en généralité à partir du cas singulier de la politique de la ville, du fait du statut particulier de cette politique et des quartiers qu'elle vise dans le débat politique français. Un exemple récent, parmi d'autres, prouve que les difficultés observées dans le cas de la politique de la ville renvoient à un problème plus structurel de légitimation du politique : la généralisation du Revenu de solidarité active (RSA). Mesure phare de la politique sociale du gouvernement, le RSA devait inaugurer une nouvelle manière d'articuler expertise et décision politique, en s'appuyant sur des expérimentations localisées accompagnées d'évaluations, lesquelles devaient permettre aux responsables politiques de décider de leur généralisation, de leur adaptation, ou de leur abandon. Alors même qu'un rapport intermédiaire du comité d'évaluation indiquait, en septembre 2008 , que les données disponibles ne permettaient pas de conclure à l'efficacité du dispositif, le gouvernement annonça son extension à l'ensemble du territoire à partir de juillet $2009 . .$.

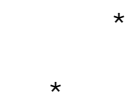

L'idée technocratique suivant laquelle il suffirait de parfaire le système d'objectifs et d'indicateurs de la LOLF pour accomplir le projet évaluatif du texte organique se heurte à des problèmes bien connus des sciences sociales. II ne suffit pas de décliner les politiques publiques en programmes, assortis d'objectifs précis et d'indicateurs de résultats pour dépasser les blocages passés de l'évaluation. La littérature anglosaxonne regorge d'exemples laissant penser que ce mode de conception et de pilotage des politiques publiques peut même aller à l'encontre de la transparence et de la performance recherchées (Beauvallet, 2009): les systèmes articulant une profusion d'objectifs et d'indicateurs, des activités de reporting systématique et des procédures d'audits réguliers produisent d'importantes distorsions dans les politiques menées, incitant les opérateurs à se concentrer sur les activités les plus « rentables », car mesurables, et non vers des tâches plus qualitatives et plus complexes à mesurer ou simplement mal renseignées par les indicateurs définis par les bailleurs de fonds (Bevan, Hood, 2006). Le souci de performance peut même contrarier la réalisation des objectifs définis par les responsables politiques. C'est notamment le cas lorsque les indicateurs définis par des tiers (hauts fonctionnaires, experts et consultants) ne reflètent pas les impacts attendus (outcomes) mais les réalisations (outputs) ${ }^{20}$, et qu'ils sont renseignés par les opérateurs eux-mêmes, ce qui ouvre la porte à toutes sortes de manipulation.

II n'est donc pas certain que le basculement de l'objet évaluatif, des politiques publiques aux programmes, constitue une réelle avancée. Cette évolution marque le triomphe d'une conception technocratique de cet exercice, au détriment d'une conception à la fois démocratique et scientifique de l'évaluation. Sur ces derniers plans, tout laisse penser qu'on assiste actuellement à une régression évaluative, mettant fin au projet d'intégration des sciences sociales -au-delà de la seule science économique- dans la conduite de l'action publique qui sous-tendait l'évaluation des politiques publiques. Les espoirs placés dans cette dernière par les sociologues et les politistes, y compris les plus critiques (Courcuff, 1993), ont d'ailleurs laissé place à des dénonciations virulentes d'une évaluation systématisée ${ }^{21}$ qui, au nom de la performance et de la transparence, fait peser une pression croissante sur les agents des services publics et leurs usagers. L'approche pluraliste qui caractérisait l'évaluation française correspondait à une forme de pari sur l'intelligence collective des acteurs publics, des usagers et des chercheurs. Elle serait remplacée par une toute autre approche, rabattant l'évaluation au rang d'instrument de contrôle, manipulé par des élites politiques et administratives qui ne cachent pas leur défiance à l'égard des bureaucraties et de leurs

\footnotetext{
${ }^{20}$ Soit un cas de figure qu'on retrouve dans la rénovation urbaine française, au sujet de laquelle on pourrait reprendre l'interrogation de Peter Ambrose dans The Politics of evaluation (2005): « Urban regeneration - Who defines the indicators ? ».

${ }^{21}$ Voir par exemple le dossier « Pour en finir avec l'évaluation » de la revue Vacarme ( $n^{\circ} 44$ - été 2008).
} 
agents. Ces critiques sont excessives, ne serait-ce que parce que l'évaluation des politiques publiques des années 1990 n'est jamais parvenue à réaliser, même partiellement, ses promesses. Mais elles ont le mérite de pointer, au-delà du décalage persistant entre la rhétorique et la pratique évaluative française, un changement d'importance: si l'évaluation ne rétroagit pas plus que par le passé sur les politiques publiques, les instruments introduits en son nom induisent de profondes transformations dans le contenu et la conduite de ces dernières. 


\section{Bibliographie}

Ambrose P. (2005) « Urban Regeneration - Who Defines the Indicators ? » in Balloch S., Taylor D (dir.) The Politics of Evaluation, Bristol : Policy Press.

Arthuis J. (2005) LOLF : culte des indicateurs ou culture de la performance ? Rapport d'information sénatorial, fait au nom de la commission des finances, Sénat : Paris.

Arkwright E., de Boissieu C., Lorenzi J.-H., Samson J. (2007) Economie politique de la LOLF. Rapport du Conseil d'Analyse Economique. Paris : La Documentation française.

Belorgey J.M. (1993) Evaluer les politiques de la ville, Conseil National des Villes.

Bénabou R., Kramarz F., Prost C. (2004) «Zones d'éducation prioritaire : quels moyens pour quels résultats ? Une évaluation sur la période 1982-1992 », Économie et statistique, 380.

Beauvallet M. (2009) Les stratégies absurdes : Comment faire pire en croyant faire mieux, Paris : Seuil

Bevan G., Hood C. (2006) «What's Measured is What Matters: Targets and Gaming in Healthcare in England », Public Administration, 84.

Bezes P. (2009) Réinventer l'Etat. Les réformes de l'administration française (1962-2008), Paris : PUF.

Bravo J. (1999) Rapport final de l'instance d'évaluation de la politique de la ville en lle-de-France, Préfecture de Région - Conseil Régional d'lle-de-France.

Chanut V. (2002) «L'évaluation: affaire d'Etat ou question d'organisation ? », Politiques et Management Public, 20, 4.

Conseil Scientifique de l'évaluation (1996) Petit guide de l'évaluation des politiques publiques, Paris : La Documentation française

Corcuff P. (1993) «Un OVNI dans le paysage français? Eléments de réflexion sur l'évaluation des politiques publiques en France », Politix, 24.

DARES (2007) «Les dispositifs de politique de l'emploi dans les zones urbaines sensibles: un accès privilégié aux CES et SIFE collectifs », Premières synthèses, premières informations, 13, 4.

Davezies L. (1999) « La discrimination positive à l'épreuve des zones d'éducation prioritaires », dact.

Deleau M., Nioche J.-P., Penz P., Poinsard R. (1986) Evaluer les politiques publiques. Méthodes, déontologie, organisation. Paris : la documentation Française.

Desrosières A. (1993) La politique des grands nombres : Histoire de la raison statistique, Paris : La Découverte.

Délégation Interministérielle à la Ville (1995) L'évaluation des contrats de ville, dossier-ressources, Paris : Editions de la DIV.

Donzelot J., Estèbe P. (1994) L'Etat animateur. Essai sur la politique de la ville, Paris : Le Seuil.

Epstein R. (2005) «Les politiques territoriales post-contractuelles: le cas de la rénovation urbaine », Politiques et Management Public,23, 3.

Duran P. (2002) «L'évaluation, au risque de son institutionnalisation », Politiques et Management Public, $20,4$.

Epstein R. (2007) « L'évaluation en développement ? Retour sur vingt ans d'évaluation de la politique de la ville » in Bouquet B., Sainsaulieu I., Jaeger M., Les défis de l'évaluation en action sociale et médicosociale, Paris : Dunod

Epstein R., Kirszbaum T. (2005) Synthèse nationale des évaluations à mi-parcours des contrats de ville 2000-2006, Rapport pour la DIV.

Estèbe P., Epstein R. (1998) Synthèse nationale des évaluations régionales et locales du Xlème Plan, Rapport pour la DIV.

Figeat D. (1981) Bilan des opérations Habitat et vie sociale menées pendant la période du Vlle Plan, Rapport pour le Commissariat Général du Plan.

Fondation Abbé Pierre (2006) L'Etat du mal logement en France, Paris : Fondation Abbé Pierre. 
Fourcade M., Le Coz G., Lejeune D., Vilchien D. (2005) Evaluation de la mobilisation des crédits de droit commun de l'Etat et contribution à l'évaluation des contrats de ville sur trois territoires, Inspection générale des affaires sociales.

Jacquier C. (2004) «Evaluation de la politique de la ville et mutations institutionnelles: un étonnant silence », Les cahiers du CRDSU, 41.

Kessler M-C., Lascoumes P., Stebon M., Thoenig J-C (dir.) (1998) Evaluation des politiques publiques, Paris : L'Harmattan.

Ion J., Peroni M., Roux J. (1996) Evaluation, évaluabilité et action publique, Rapport pour le Commissariat Général du Plan.

Lelévrier C. (2008) Mobilités et trajectoires résidentielles des ménages relogés lors des opérations de renouvellement urbain. Synthèse de travaux menés entre 2004 et 2007, rapport PUCA, DIV, DREIF.

Levy F. (1988) Bilan / perspectives des contrats de plan de développement social des quartiers, Paris : la Documentation française.

Monnier E., Henard F. (2001) «L'évaluation des politiques publiques dans les collectivités territoriales: exercices imposés et initiatives propres », Pouvoirs Locaux, 51.

Kirszbaum T. (1998) Fonction de coordination des services de l'Etat et contractualisation dans la politique de la ville. Essai de synthèse des évaluations produites dans le cadre du Xlème Plan, Rapport pour le Plan Urbain.

Linhart V. (1996) La "ville" comme objet de politique publique. Genèse et institutionnalisation de la politique de la ville en France. Thèse de doctorat, IEP Paris.

Maury S. (2008) «La LOLF est-elle un bon moyen d'évaluer les politiques publiques ? ", Actualité Juridique Droit Administratif, 25.

Nioche J-P., Poinsard R. (1984) L'évaluation des politiques publiques. Paris : Economica

Perret B. (2008) «L'évaluation des politiques publiques. Entre culture du résultat et apprentissage collectif », Esprit, décembre.

Perret B. (2001) L'évaluation des politiques publiques, Paris : La Découverte.

Rathelot R., Sillard P., 2007. «Zones Franches Urbaines : quels effets sur l'emploi salarié et les créations d'établissements ? », INSEE, Document de travail G2007/11.

Robert C. (2005) «L'ANRU au risque de l'évaluation. Que va-t-on réellement évaluer et quand va-t-on s'y mettre ? ", Recherche sociale, 176.

Schneider A.L., Ingram H. (1997) Policy design for democracy, Lawrence : University Press of Kansas.

Siné A. (2006) L'ordre budgétaire : L'économie politique des dépenses de l'Etat. Paris : Economica.

Siné A., Veillet I. (2007) «La performance, un outil et une démarche indispensables au pilotage des politiques publiques », Regards sur l'actualité, 335.

Spenlehauer V., Warin P. (2000) «L'évaluation au services des conseils régionaux », Sociologie du travail, $42,2$.

Stufflebeam D.L. (2001) « The metaevaluation imperative », The American Journal of Evaluation, 22, 2.

Thoenig J-C. (2002) «L'évaluation en actes : leçons et perspectives », Politiques et Management Public, 20,4 .

Tréguer C (2002) Les politiques publiques favorisent-elles les quartiers pauvres? Thèse de doctorat, Institut d'Urbanisme de Paris.

Vacarme (2008) « Dossier : Pour en finir avec l'évaluation », 44.

Viveret P. (1989) L'évaluation des politiques et des actions publiques, Paris : la documentation Française.

Weaver R.K. (1986) « The Politics of Blame Avoidance », Journal of Public Policy, 6,4. 